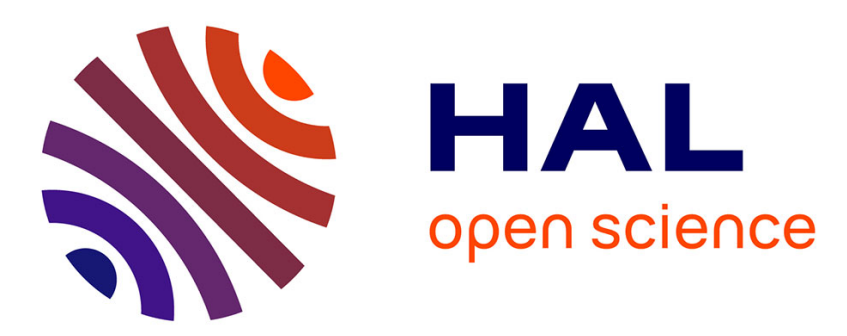

\title{
Reynolds Number Effects on Statistics and Structure of an Isothermal Reacting Turbulent Wall-Jet
}

Zeinab Pouransari, Luc Vervisch, Arne V Johansson

\section{To cite this version:}

Zeinab Pouransari, Luc Vervisch, Arne V Johansson. Reynolds Number Effects on Statistics and Structure of an Isothermal Reacting Turbulent Wall-Jet. Flow, Turbulence and Combustion, 2014, 92 (4), pp.931-945. 10.1007/s10494-014-9539-3 . hal-01612426

\section{HAL Id: hal-01612426 https://hal.science/hal-01612426}

Submitted on 29 Oct 2018

HAL is a multi-disciplinary open access archive for the deposit and dissemination of scientific research documents, whether they are published or not. The documents may come from teaching and research institutions in France or abroad, or from public or private research centers.
L'archive ouverte pluridisciplinaire HAL, est destinée au dépôt et à la diffusion de documents scientifiques de niveau recherche, publiés ou non, émanant des établissements d'enseignement et de recherche français ou étrangers, des laboratoires publics ou privés. 


\title{
Reynolds Number Effects on Statistics and Structure of an Isothermal Reacting Turbulent Wall-Jet
}

\author{
Zeinab Pouransari · Luc Vervisch • Arne V. Johansson
}

\begin{abstract}
Three-dimensional direct numerical simulation (DNS) is used to investigate the effects of changing the Reynolds number on dynamics of a reacting turbulent wall-jet. The flow is compressible and a single-step isothermal global reaction is considered. At the inlet, fuel and oxidizer enter the domain separately in a non-premixed manner. In this study, the bulk Reynolds number of the flow, in terms of the inlet quantities, varies from $R e=2000$ to $R e=6000$, which results in a comparable change in friction Reynolds numbers. The DNS database in Pouransari et al. (Phys. Fluids 23(085104), 2011) is used for the lower Reynolds number case and for the higher Reynolds number case, a new DNS is performed. One of the main objectives of this study is to compare the influences of changing the Reynolds number of the isothermal flow with the heat-release effects caused by the chemical reaction, that we studied earlier in Pouransari et al. (Int. J. Heat Fluid Flows 40, 65-80, 2013). While, both turbulent and flame structures become finer at the higher Reynolds number, the effect of decreasing the Reynolds number and adding the combustion heat release are compared with each other and found to be similar for some aspects of the flow, but are not always the same.
\end{abstract}

Keywords Reynolds number effects · Turbulent · Combustion · Mixing scales · Wall-jet

\section{Introduction}

Many numerical studies have been performed on various aspects of turbulent combustion in several flow configurations. However, in most of these studies, in particular when direct

\footnotetext{
Z. Pouransari $(\bowtie) \cdot A$. V. Johansson

Linné FLOW Centre, KTH Mechanics, SE-100 44 Stockholm, Sweden

e-mail: zeinab@mech.kth.se

L. Vervisch

CORIA - CNRS and INSA de Rouen, Technopole du Madrillet, BP 8,

76801 Saint-Etienne-du-Rouvray, France
} 
numerical simulation (DNS) is employed as the computational tool, the Reynolds number of the flow is very limited and much lower than in real physical situations. Thus, some of the conclusions drawn in these cases may be subject to both qualitative and quantitative changes at higher Reynolds numbers.

Effects of Reynolds number in canonical flows have been addressed both numerically and experimentally, for turbulent channel flows [9, 13, 24], turbulent boundary layers $[5,21]$ and pipe flows $[7,11]$, including studies on the scaling properties. Today, the Reynolds number of experimental facilities has increased substantially and both smooth and rough wall flows are still of great interest, see [2, 12]. It is only in the last few years that DNS studies have reached Reynolds numbers where significant scale separation (between inner and outer scales) can be claimed.

Many other efforts in the study of the Reynolds number effects are directed towards the degree of local isotropy and the fine structures of turbulence. For these purposes, experiments and numerical investigations with different Reynolds numbers are carried out and in general at higher Reynolds numbers a high degree of universality of small scales and local isotropy have been found, see [8, 22, 25].

Reynolds number effects on scalar dissipation rate transport and its modeling in turbulent premixed combustion are addressed in [4]. Scaling arguments have been used to explain the Reynolds number effects on the turbulent transport, scalar turbulence interaction and the combined reaction and molecular dissipation terms. It was found that the modeling of the term arising from density variation has no significant Reynolds number dependence (within the range examined).

Although DNS is a reliable tool for the study of various turbulent flows. However, some cases such as compressible and reacting flows are computationally more demanding and hence numerical studies of turbulent combustion flows are usually restricted to lower Reynolds number ranges [15, 23].

In our previous studies of wall-jet flows, we have addressed the turbulent flow effects on the structure of the flame both in the absence and presence of combustion heat-release $[16,18]$. However, in those studies, we kept the bulk Reynolds number of the flow constant and focused on the heat-release and turbulence interactions. In the present study, the bulk Reynolds number is increased by a factor of three, which results in a comparable increase in the friction Reynolds number.

In the present investigation, the Reynolds number effects in a reacting turbulent wall-jet are studied. In particular, we are interested in comparing these effects, which we observed in the study of the heat-release effects at the higher Reynolds number where the scale separation starts to become significant. In general, including the combustion heat-release in a reacting turbulent flow decreases the local Reynolds number. Thus, in our previous studies on the effects of heat-release in a reacting turbulent wall-jet; we observed different changes in the mixing scales of the flow. There, we examined how the presence of heat-release could affect the scales of turbulence and in return how it can affect the global reaction. Here, we disregard the heat-release effects, and instead change the bulk Reynolds number of the flow. Therefore, we can study the Reynolds number effects on the isothermal reaction and on the structure and statistics of the flow in the absence of thermal effects.

This paper is organized as follows. The governing equations are shown in Section 2 and the numerical method and the flow configuration are explained in Section 3. The Reynolds number effects on both the turbulent structures and the flame characteristics are included in Section 4, followed by the summary and conclusions in Section 5. 


\section{Governing Equations}

The conservation equations of total mass, momentum and energy in the compressible form read

$$
\begin{aligned}
\frac{\partial \rho}{\partial t}+\frac{\partial \rho u_{j}}{\partial x_{j}} & =0, \\
\frac{\partial \rho u_{i}}{\partial t}+\frac{\partial \rho u_{i} u_{j}}{\partial x_{j}} & =-\frac{\partial p}{\partial x_{i}}+\frac{\partial \tau_{i j}}{\partial x_{j}}, \\
\frac{\partial \rho E}{\partial t}+\frac{\partial \rho E u_{j}}{\partial x_{j}} & =-\frac{\partial q_{i}}{\partial x_{i}}+\frac{\partial\left(u_{i}\left(\tau_{i j}-p \delta_{i j}\right)\right)}{\partial x_{j}} .
\end{aligned}
$$

Here $\rho$ is the total mass density, $u_{i}$ are the velocity components, $p$ is the pressure and $E=e+\frac{1}{2} u_{i} u_{i}$ is the total energy. The summation convention over repeated indices is used and $\delta_{i j}$ is the Kronecker delta. The heat fluxes are approximated by Fourier's law according to $q_{i}=-\lambda \frac{\partial T}{\partial x_{i}}$, where $\lambda$ is the coefficient of thermal conductivity and $T$ is the temperature. The viscous stress tensor is defined as $\tau_{i j}=\mu\left(\frac{\partial u_{i}}{\partial x_{j}}+\frac{\partial u_{j}}{\partial x_{i}}\right)-\mu \frac{2}{3} \frac{\partial u_{k}}{\partial x_{k}} \delta_{i j}$ where $\mu$ is the dynamic viscosity. The fluid is assumed to be calorically perfect and to obey the ideal gas law according to $e=c_{v} T, \quad p=\rho R T$, and a ratio of specific heats of $\gamma=c_{p} / c_{v}=1.4$ is used. The viscosity is determined through Sutherland's law $\frac{\mu}{\mu_{j}}=\left(\frac{T}{T_{j}}\right)^{3 / 2} \frac{T_{j}+S_{0}}{T+S_{0}}$, where $T$ is the local temperature, $T_{j}$ is the jet center temperature at the inlet and $S_{0}$ is a reference value, taken as $S_{0}=110.4 \mathrm{~K}$. The temperature dependent viscosity enables the simulation to better capture the small compressibility effects.

Conservation of the species masses is governed by

$$
\frac{\partial \rho \theta_{k}}{\partial t}+\frac{\partial}{\partial x_{j}}\left(\rho \theta_{k} u_{j}\right)=\frac{\partial}{\partial x_{j}}\left(\rho \mathscr{D} \frac{\partial \theta_{k}}{\partial x_{j}}\right)+\dot{\omega}_{k},
$$

where $\theta_{k}$ and $\dot{\omega}_{k}$ are the mass fractions and the reaction rate of the oxidizer, fuel and passive scalar species. An equal diffusion coefficient $\mathscr{D}$ for all scalars is used to approximate the diffusive fluxes.

The simulated reaction is modeled by a single-step irreversible reaction between oxidizer species $O$ and fuel species $F$ that react to form a product $P$, which is described as $v_{O} O+$ $v_{F} F \longrightarrow v_{P} P$. Stoichiometric coefficients of one are used for all species and the molecular weights are also assumed to be equal for the reactants. The reaction mass rate is formulated as $\dot{\omega}=k_{r} \rho^{2} \theta_{o} \theta_{f}$. The reaction rate is prescribed by the Damköhler number as

$$
D a=\frac{\tau_{\text {conv }}}{\tau_{\text {react }}}=\frac{h}{U_{j}} k_{r} \rho_{j},
$$

where $k_{r}$ is the reaction rate and $U_{j}$ and $\rho_{j}$ are the centerline velocity and the density of the jet at the inlet. In both of the simulation cases a constant global Damköhler number with a value of $D a=3$ is used. No heat release is used in the present simulation and only the oxidizer and fuel mass fractions are computed since the product does not enter in the reaction rate expression. Since the flow is uncoupled from the reactions, the influence of increasing the Reynolds number can be studied in the absence of temperature effects. Apart from reacting species, a passive scalar equation is also solved for comparison. 


\section{Numerical Method and Parameters}

A fully compressible Navier-Stokes solver is employed for simulation of the wall-jet. The code uses a sixth-order compact finite difference scheme for spatial discretization and a third-order Runge-Kutta method for the temporal integration.

The computational domain is a rectangular box of size $\left[L_{x} \times L_{y} \times L_{z}\right]$ where $L_{x}, L_{y}$ and $L_{z}$ denote dimensions in the streamwise, wall-normal and spanwise directions, respectively, and are expressed in terms of the inlet jet height $h$ in Table 1 . The number of grid points are $\left[N_{x} \times N_{y} \times N_{z}\right]$ and the inlet based Reynolds number of the wall-jet is $R e=6000$. Hereafter, this case is referred to as case II, while the DNS data in paper [16] is tagged as case I, and its grid points and the Reynolds number are also given in Table 1. The bulk Reynolds number of case II is increased by a factor of three and a corresponding grid of 180 million cells is employed. The density and temperature are varied over the jet profile and the wall temperature is kept constant and equal to the ambient flow temperature. The specifications of the simulations are summarized in Table 1.

A Taylor micro scale in the $i^{\text {th }}$ direction can be defined as

$$
\lambda_{g, i}=\overline{\frac{15 v}{\epsilon} u_{i, r m s}^{2}},
$$

where $\epsilon$ is the viscous dissipation rate. The Kolmogorov length scale is defined as

$$
\eta={\frac{v^{3}}{\epsilon}}^{1 / 4} \text {. }
$$

The Taylor micro scale is considered as the "smallest large scale", which is responsible for the dissipation of energy, see [3], whereas the Kolmogorov scale is the smallest turbulence length scale and is associated with the viscous dissipation of turbulent kinetic energy.

Figure 1 shows these scales in the current simulations. In this figure a dashed line is used for case II with the higher Reynolds number and a solid line for case I with the lower Reynolds number. Note that these line styles are used throughout the paper unless otherwise stated. From Fig. 1a and b, we can observe that the small scales of the flow are smaller at almost all wall normal levels for the higher Reynolds number case, however, moving further away from the wall this reduction in the scale sizes is even more accentuated. An important point to notice is how Kolmogorov scale compares to the grid spacing in the vertical direction. The jet flame burns mostly below $y / h=4$, where most of the flameturbulence interactions also take place. In this region, for both cases, the grid spacing is either smaller than the Kolmogorov scale or in order of that. For case II, with the higher Reynolds number the resolution is finer. In the axial direction, (figure not shown) a same trend is observed and increasing the Reynolds number decreases the $\lambda_{x}$ in general and also the grid spacing is comparable with the Kolmogorov scale. However, close to the transition region, $\lambda_{x}$ reaches a maximum value in both cases at about the same location, where the Reynolds number effects on size of the small scales is minimum.

Table 1 Parameters of direct numerical simulations of reacting turbulent wall jet flows; $\operatorname{Pr}=0.7, S c=0.7$ and $D a=3$ in both of the simulations

\begin{tabular}{lllllll}
\hline Case & $R e$ & $L_{x} \times L_{y} \times L_{z}$ & $N_{x} \times N_{y} \times N_{z}$ & $\Delta x^{+}$ & $\Delta y_{1}^{+}$ & $\Delta z^{+}$ \\
\hline I & 2000 & $35 \times 17 \times 7.2$ & $320 \times 192 \times 128$ & $10.4 \sim 12.99$ & $0.89 \sim 11.93$ & $5.1 \sim 6.3$ \\
II & 6000 & $35 \times 14 \times 7.2$ & $960 \times 480 \times 384$ & $7.4 \sim 11.2$ & $0.83 \sim 11.49$ & $4.9 \sim 6.9$ \\
\hline
\end{tabular}


(a)

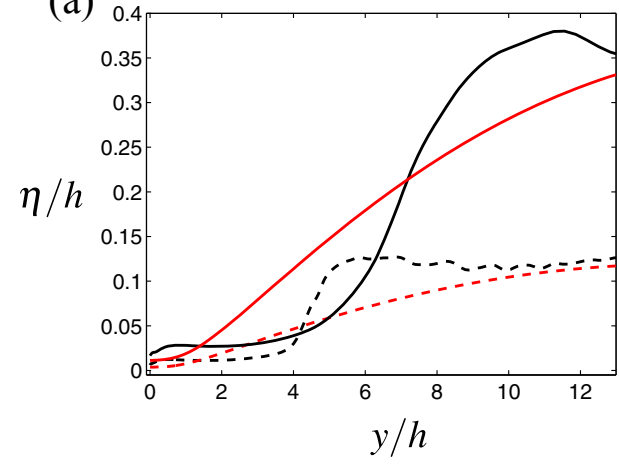

(b)

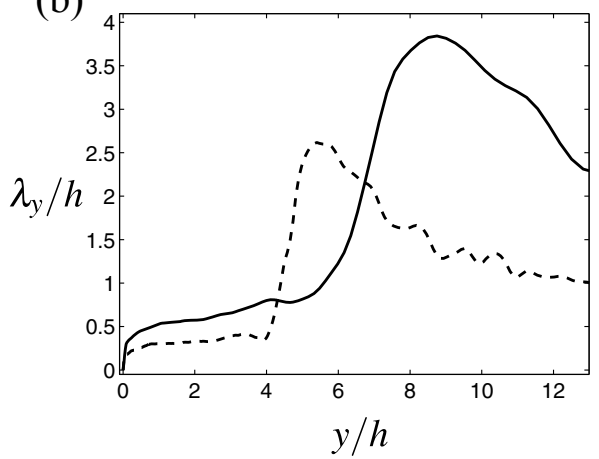

Fig. 1 The Kolmogorov length scale $\eta$ and the Taylor micro scale $\lambda_{y}$ versus the computational grid spacing $\Delta$ in the $y$-direction at downstream position $x / h=25 ; \mathbf{a} \eta$ and $\mathbf{b} \lambda_{y}$; solid line: case I $(R e=2000)$ and dashed line: case II $(R e=6000)$. Red lines show the computational grid spacing $\Delta_{y} / h$ and solid line for case I and red dashed line for case II

\section{Results and Discussions}

\subsection{Turbulent statistics and flow structure}

To get a general insight into the two cases with different bulk Reynolds number, we examine the friction Reynolds number $R e_{\tau}=u_{\tau} \delta / v_{w}$; see Fig. 2a. When an appropriate outer length-scale $\delta$ is used, the friction Reynolds number can be seen as an estimate of the outerlayer to inner-layer length-scale ratio. Around $x / h=25$, for case II $(R e=6000)$ the friction Reynolds number reaches the value of $R e_{\tau}=540$ and for case I $(R e=2000)$ it reaches $R e_{\tau}=220$. Thus the effective Reynolds number based on viscous scaling is almost two and a half times larger for case II. In this paper, most of the statistics are taken at downstream position $x / h=25$, where it is well inside the fully developed region for both cases.

The accurate experimental determination of the skin friction still remains a major challenge in turbulent wall-bounded flows. In flows such as in the reacting turbulent wall-jet flow, the skin friction drag is one of the factors that are responsible for the overall performance efficiency. Thus, different methods of drag reductions are considered in these devices. The downstream development of the skin friction coefficient, $C_{f}=2\left(u_{\tau} / U_{m}\right)^{2}$ is shown in Fig. 2b for cases I and II. In the fully turbulent region at $x / h=25, C_{f}$ is about $40 \%$ lower for the higher Reynolds number case. These observations can, in some sense, be compared with the reductions in $C_{f}$ observed when heat release effects were added (to the lower $R e$ case) and hereby further reduced with the Reynolds number, see [17] for further discussions about the drag reduction.

The mean turbulence intensity for the three components of the velocity is plotted in Fig. 3a-c using semi-local inner scaling. The turbulent kinetic energy is also plotted in Fig. $3 \mathrm{~d}$ using the same normalization. With this scaling we observe a good collapse in the near-wall region, up to $y^{*}=7 \sim 8$, where $y^{*}=\left(\bar{\mu} / \bar{\mu}_{w}\right) /\left(\bar{\rho} / \bar{\rho}_{w}\right) y^{+}$. In order to make the comparison easier, the same scaling method is used later for plotting the two-dimensional spectra. For details of this normalization see [18]. Figure 3a shows that increasing the Reynolds number will increase the fluctuation intensities in the streamwise direction both in the inner and outer regions. There is a clear increase in the amplitude of the two peaks 

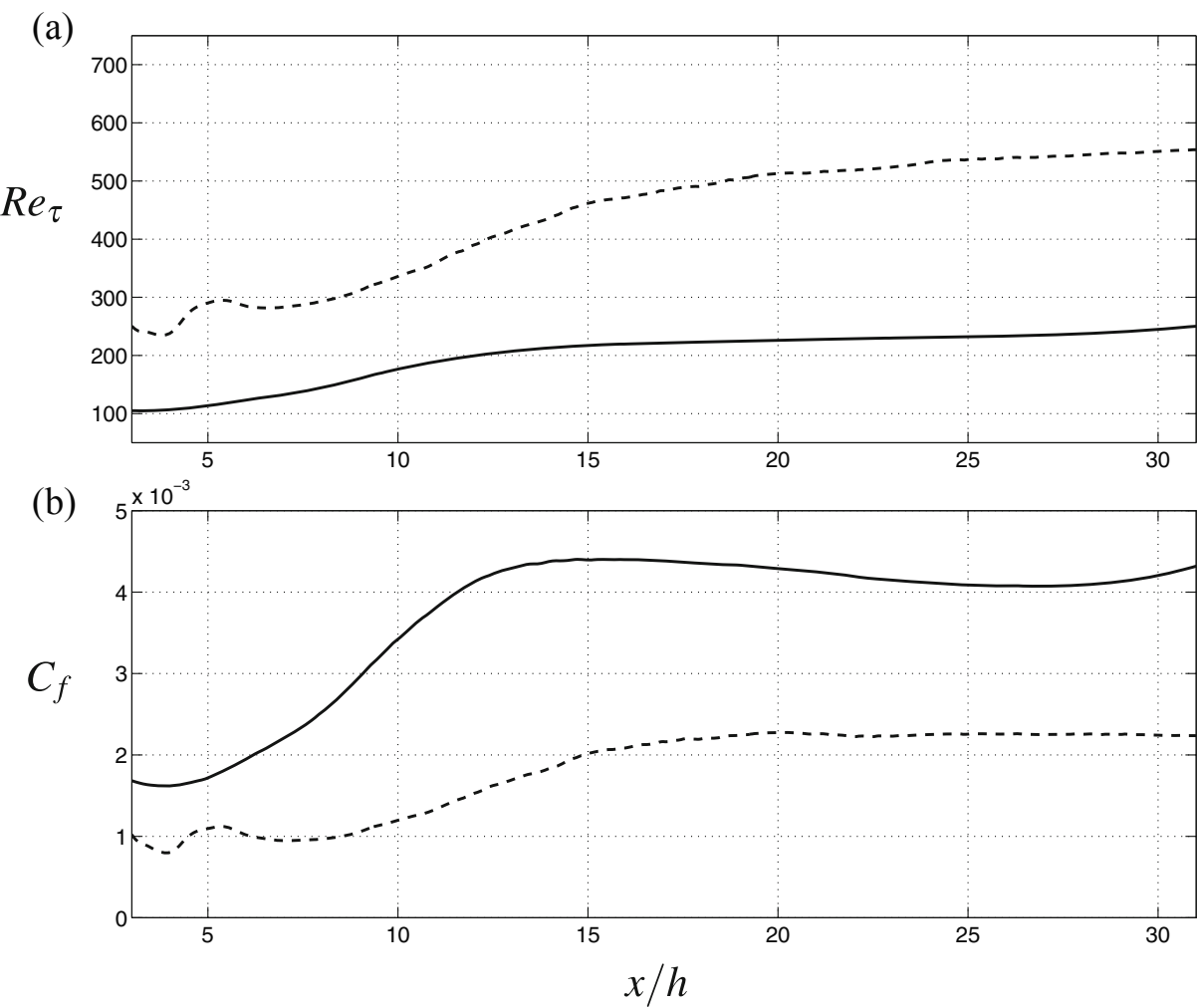

Fig. 2 a Downstream development of the friction Reynolds number; b Downstream development of the skin friction coefficient; and again solid line for case I $(R e=2000)$ and dashed line for case II $(R e=6000)$

of the fluctuation intensity profile and also as a sign of increase in the Reynolds number they get further separated from each other. Both simulations show that $u_{r m s}^{\prime \prime} / u_{\tau}^{*}$ reaches a maximum value around $y^{+}=10 \sim 12$, and has a second peak due to the shear layer of the jet flow. The $u_{r m s}^{\prime \prime} / u_{\tau}^{*}$ profiles exhibit a clear Reynolds-number dependence for $y^{+}>10$. The near wall turbulence intensity peak becomes broader and the peak value increases with increasing the Reynolds number. This is similar to what is observed in turbulent channel flow; see [24].

For the spanwise component, Fig. 3c, the peak of the fluctuation profile slightly increases with increasing of the Reynolds number and also moves further away from the wall. Figure $3 \mathrm{~b}$ shows that the wall-normal velocity fluctuation levels in some regions decreases when the Reynolds number increases, this behavior is the same if the absolute values are considered without any scaling and signifies the development of a plateau in the intermediate overlap region. The peak value is similar for the two Reynolds numbers considered. However, what is consistent using different scalings, is that due to increasing the Reynolds number, the outer maximum is shifted further outward from the wall region and the locations of the two peaks get further separated from each other for all components of the fluctuations. As a result, the same trend is observed in the turbulent kinetic energy profile (Fig. 3d). The advantage of using the semi-local scaling is the good collapse of the profiles in the near-wall region for the two cases with different Reynolds numbers. 

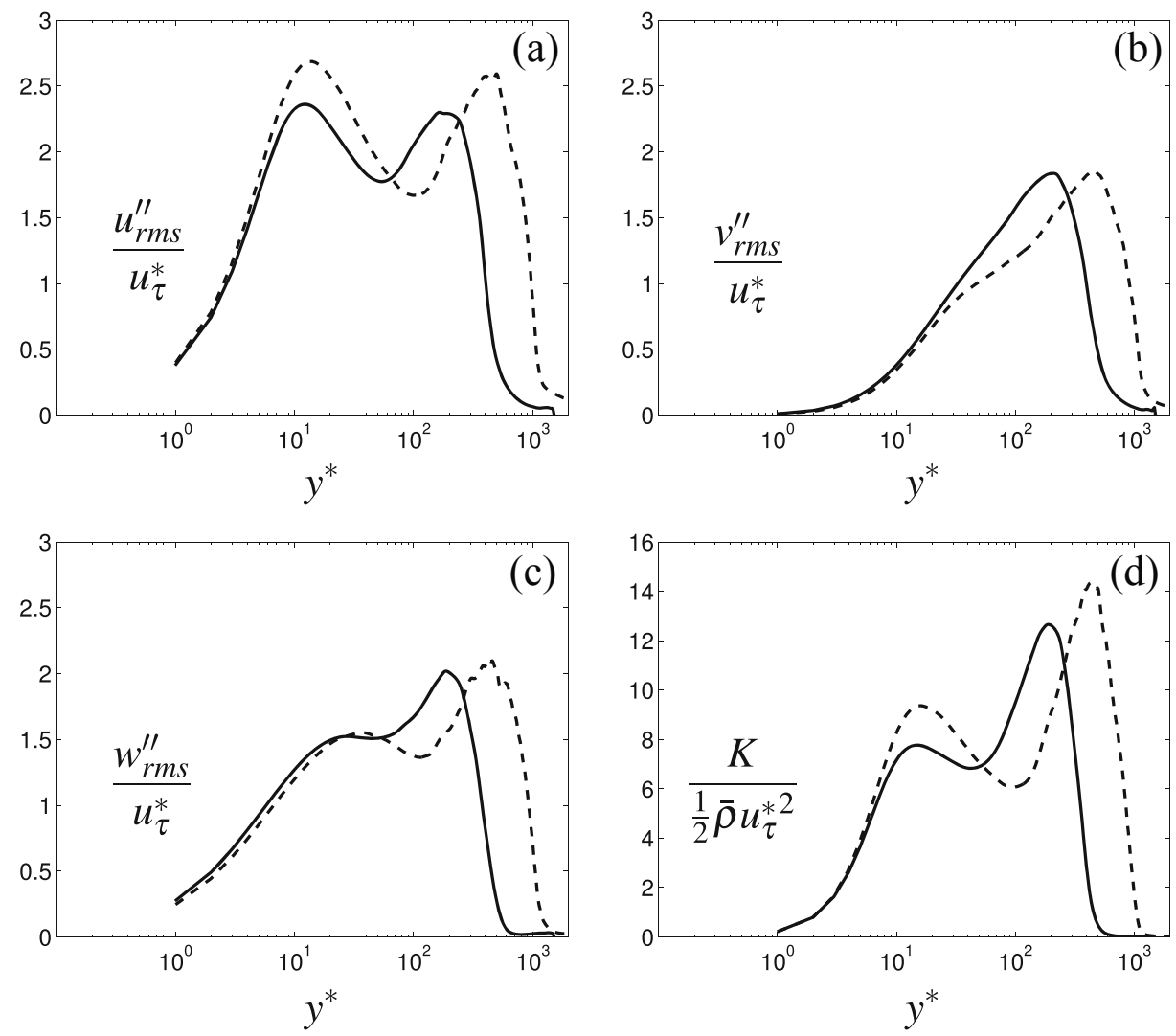

Fig. 3 a Streamwise, b wall-normal, c spanwise fluctuation intensity and $\mathbf{d}$ mean turbulent kinetic energy using semi-local normalization defined as $u_{\tau}^{*}=\sqrt{\bar{\rho}_{w} / \bar{\rho}} u_{\tau}$ and $l^{*}=\frac{(\bar{\mu} / \bar{\rho})}{u_{\tau}^{*}}=\frac{\left(\bar{\mu} / \bar{\mu}_{w}\right)}{\bar{\rho} / \bar{\rho}_{w}} l^{+}$, where $u_{\tau}=$ $\sqrt{\tau_{w} / \bar{\rho}_{w}}$ and $l^{+}=\bar{v}_{w} / u_{\tau}$ are the friction velocity and length scales, and wall conditions are denoted by a subscript $w$. Profiles are at $x / h=25$ and line styles are as in Fig. 2

The viscous dissipation rate, $\epsilon=\overline{\tau_{i j} \frac{\partial u_{i}^{\prime \prime}}{\partial x_{j}}} / \bar{\rho}$, is presented in Fig. 4. Inner scaling is employed using $u_{\tau}^{4} / \nu$, and correspondingly $\left(U_{m}-U_{c}\right)^{3} / y_{1 / 2}$ is used for outer scaling. The near-wall values of $\epsilon$ in terms of wall units agree reasonably well with values found in turbulent channel flows. In the outer shear layer, maxima are observed slightly inside the half-height, coinciding with the position of the Reynolds stress maxima. This behavior is similar to that of the non-reacting turbulent wall-jet simulations $[1,16]$.

To identify and explain the influences of Reynolds number effects on the structure of the turbulent wall-jet and to have a general idea about the flow field, the streamwise velocity fluctuations are visualized in Fig. 5 and indicate that the jet is fully turbulent beyond $x / h \approx 15$. Figure 5 a shows the instantaneous fluctuations of the streamwise velocity in the $x z$-plane at $y^{+} \approx 8$. Elongated streamwise streaks, typically present in the viscous sub-layer of wall-bounded flows are also seen in the turbulent wall-jets presented here. The distance between the streaks is roughly independent of the Reynolds number when scaled with wall units [19]. 

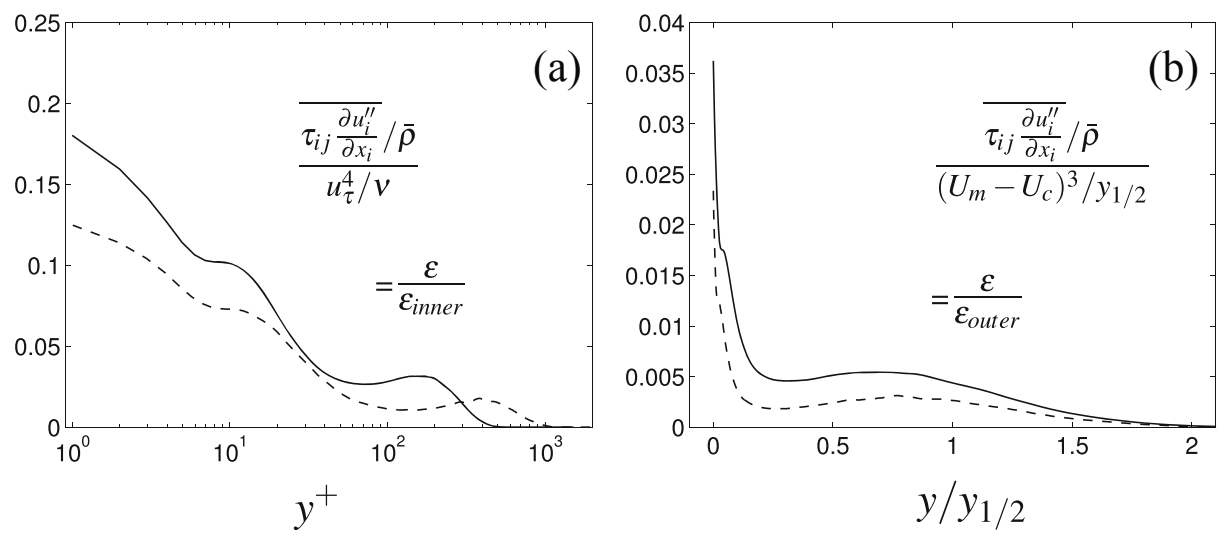

Fig. 4 Turbulent kinetic energy viscous dissipation rates, using $\mathbf{a}$ inner and $\mathbf{b}$ outer scalings. Profiles are at $x / h=25$ and line styles are as in Fig. 2

The turbulent flow structure near the walls is significantly different at a higher Reynolds number compared to the lower Reynolds number. For instance, the hairpin and vorticity structures and their interactions will be evolved and modified going from low Reynolds number to high Reynolds number cases. Though, for the range of Reynolds numbers considered in this study, the general form of the structures remain fairly similar between the two cases. However, the range of scales increases in proportion to the Reynolds number. To further illustrate the range of spanwise scales near the wall, one-dimensional spectra at $y^{+}=8$ are shown in Fig. 6a for the three velocity components (in outer units). As we should expect, one can observe an increase in the range of scales that is essentially proportional to the increase in Reynolds number. The spectrum of the streamwise velocity is clearly influenced at all wave numbers. The general increase of the turbulence energy seen in Fig. $3 \mathrm{~d}$, is also
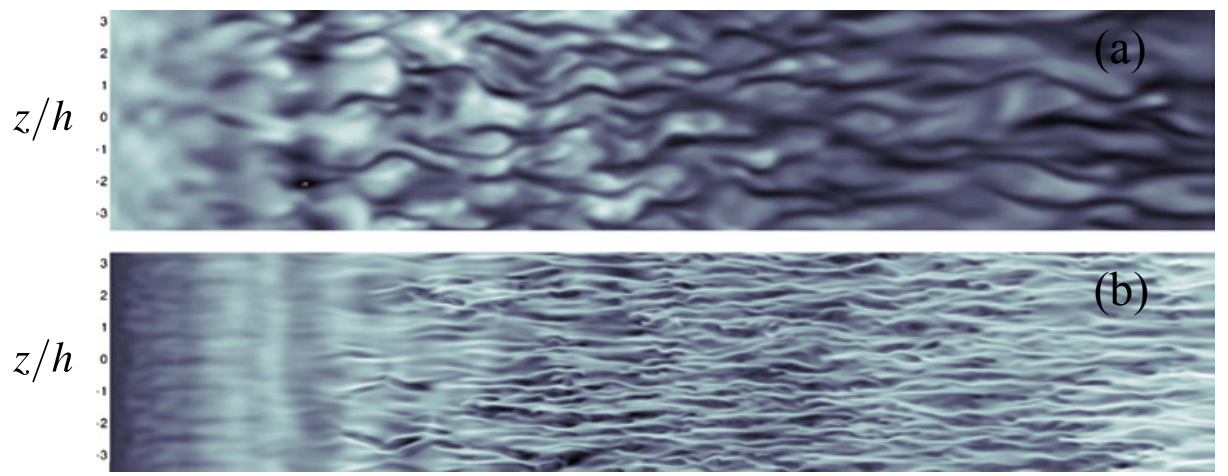

$$
x / h
$$

Fig. 5 Instantaneous snapshots of the streamwise velocity fluctuations at $y^{+} \approx 8$ for $\mathbf{a}$ case I and $\mathbf{b}$ case II; Light and dark colors represent positive and negative fluctuations 

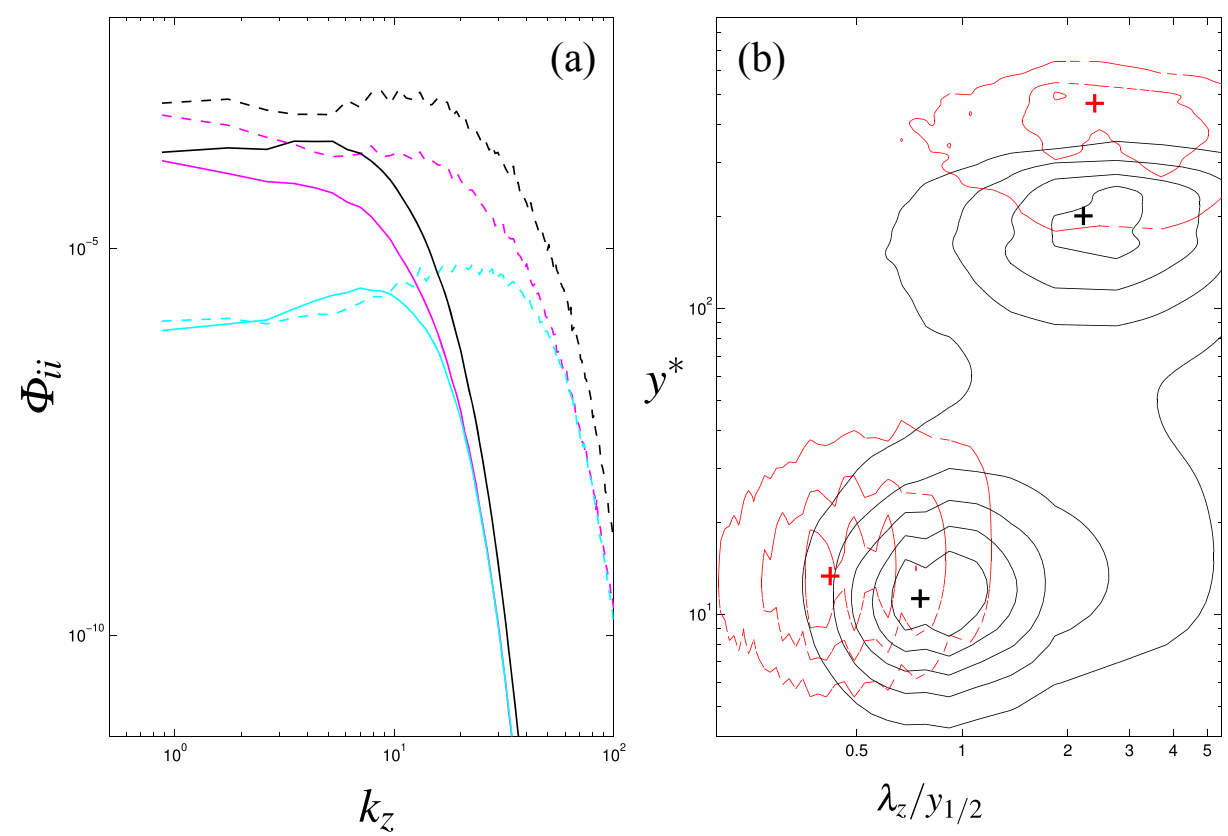

Fig. 6 a One-dimensional spanwise spectra, $\Phi_{i i}$, at $y^{+}=8$. Color code as follows, streamwise: $\Phi_{11}$ (black), wall-normal: $\Phi_{22}$ (cyan) and spanwise: $\Phi_{33}$ (magenta); b Premultiplied spanwise spectra, $E_{u u}^{z}{ }^{*}=\lambda_{z} \Phi_{u u} / u_{\tau}^{* 2}$, of the streamwise velocity fluctuations, case I (black, solid) and case II (red, dashed), scaled in the semi-local inner scaling; Contours are at $x / h=25$

reflected in the spectra. Hence, increasing the Reynolds number has affected different components in an anisotropic manner. While the energy content at low wave numbers both for the streamwise and spanwise components is substantially increased, it is almost unaffected for the wall-normal component.

A more comprehensive insight can be gained by examining the two-dimensional spectra, see $[6,20]$. The two-dimensional premultiplied spectra, $E_{u u}^{z}=\lambda_{z} \Phi_{u u} / u_{r m s}^{2}$ of the streamwise velocity fluctuations as a function of spanwise wavenumber and wall-normal position are shown in Fig. 6b, in inner units, for the two cases. Here, we have used the semi-local inner scaling as, $E_{u u}^{z}{ }^{*}=\lambda_{z} \Phi_{u u} / u_{\tau}^{* 2}$; A corresponding wavelength, as $\lambda_{z}^{*}=\left(2 \pi / \kappa_{z}\right) u_{\tau}^{*} / \nu$, could not be defined, due to the density variation along the wall-normal axis, see [18]. Here, $\lambda_{z}=2 \pi / \kappa_{z}$ is the wavelength and is scaled with the corresponding local half-height of the jet for each case. The spectra have two peaks. The inner peak, which corresponds to the near-wall streaks, is located almost at the same wall-distance, in terms of semi-local inner scaling $\left(y^{*}\right)$ for the two cases. The outer-peak, which corresponds to the large energetic structures of the outer-layer, is located at different distances from the wall for the two cases, when inner scaling is used. This behavior is consistent with the observations made in the profiles of the turbulent kinetic energy.

The peaks would essentially coincide for outer scaling $\left(y / y_{1 / 2}\right)$. The figure confirms that the inner peak location is shifted to smaller scales. Though, $\lambda_{z}^{*}$ cannot be used for the entire figure, one can estimate the location of the peak in terms of $\lambda_{z}^{*}$ to be roughly the same for the two cases. In outer scaling $\lambda_{z} / y_{1 / 2}$, the outer peak for the higher Reynolds number is only slightly shifted to higher values. 


\subsection{Scalar statistics and flame structure}

Snapshots of the reaction term for the two cases are presented in Fig. 7. These plots show that the reaction mainly occurs in the upper shear layer in thin sheet-like structures, but also takes place near the wall. Figure 7 confirms that the flame gets much more wrinkled as the Reynolds number increases. The structure of the flame is affected both in the outer layer, close to the half-height of the jet, and in the near-wall region. Even though that the flame gets much more wrinkled and folded and convoluted in different regions, in general it also gets thinner and becomes more intensified and concentrated. This reduction in the thickness with increasing the Reynolds number is expected.

The downstream developments of the fuel and oxidizer mass fluxes are plotted in Fig. 8a, which shows that the fuel species are consumed faster at the lower Reynolds number case. At $x / h=30$ for example about $40 \%$ less fuel is consumed in the higher Reynolds number case compared to case I. Thus, increasing the Reynolds number decreases the rate of fuel consumption. Moving further downstream, the oxidizer mass flux in both cases increases after $x / h=17$, as a result of the oxidizer influx at the top of the domain.

The wall-normal growth can usually also be characterized by the development of the half-height of the jet, which is the position in the outer shear layer, where the velocity is equal to half its maximum excess value. Previous studies of turbulent wall-jet flows, show a linear half-height growth for the wall-jet, which is about $20 \sim 30 \%$ lower than that of plane jets, see [14]. Downstream development of the half-height of the wall-jet is shown in Fig. $8 \mathrm{~b}$ for the two cases. In the higher Reynolds number case II, the jet half-height growth is decreased, which is in accordance with the decrease in the length scales shown in Fig. 1 and a relative shift of energy content to smaller scales.

The scalar dissipation rate is a quantity of prominent importance in non-premixed combustion. The Reynolds number effects on the scalar dissipation rate are illustrated in Fig. 9, where the iso-contours of the scalar dissipation rate for two cases are shown. The fields are plotted on a logarithmic scale to accentuate their wide dynamic range. In both cases, long thin sheet-like structures are observed, similar to what is reported in [10]. These figures
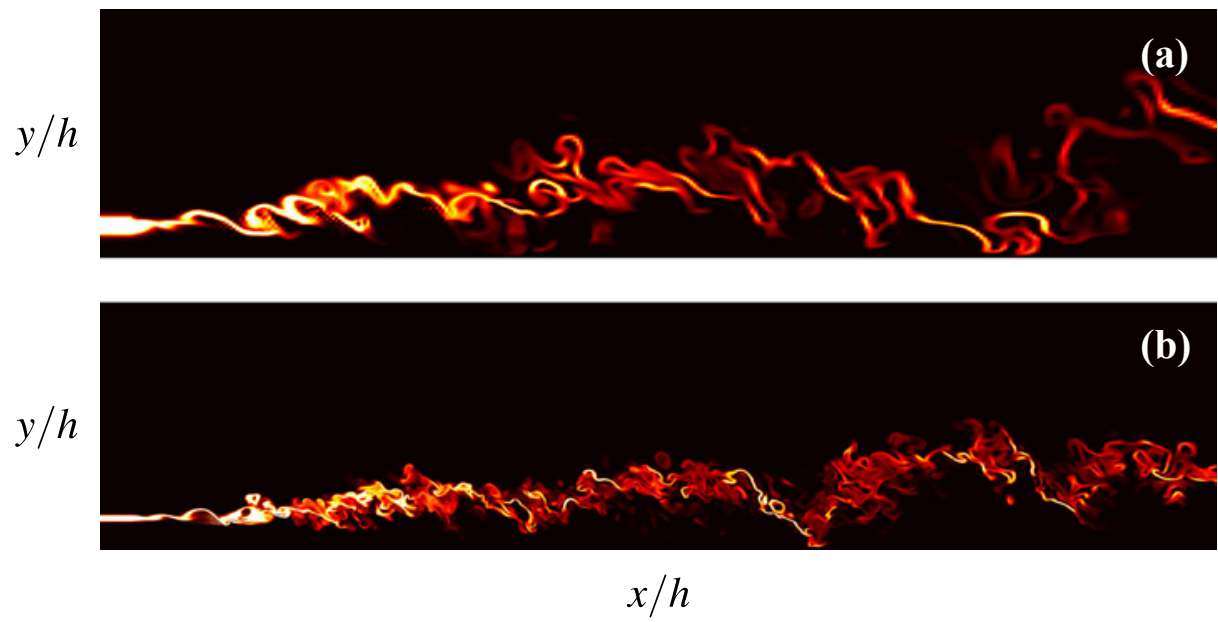

Fig. 7 Instantaneous snapshots of the reaction rate fields for $\mathbf{a}$ case I and $\mathbf{b}$ case II; The lighter color indicates higher reaction rates 

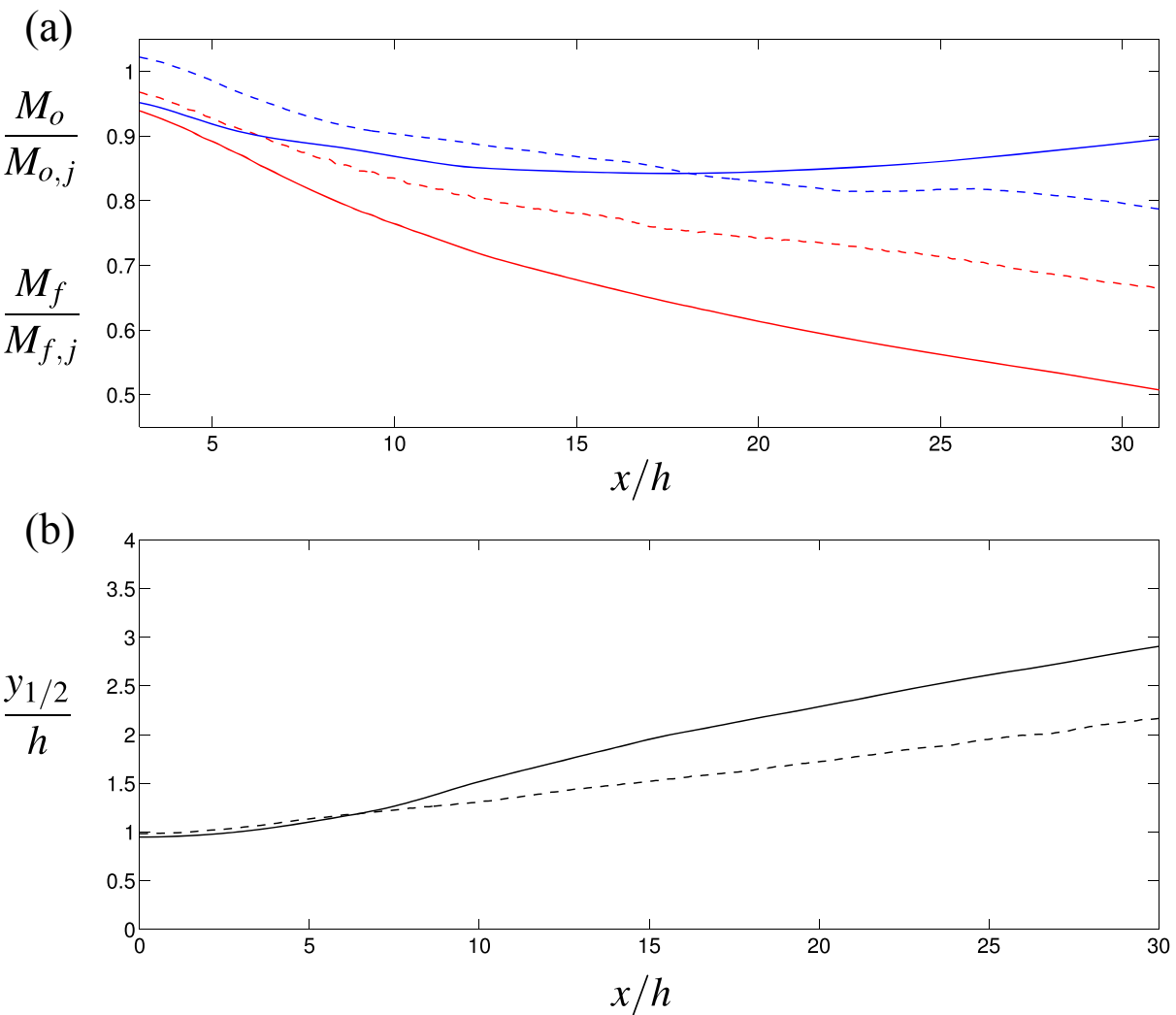

Fig. 8 Downstream development of a the reactants mass flux, red line is used for fuel and blue for oxidizer; b the half-height of the wall-jet, Profiles are at $x / h=25$ and line styles are as in Fig. 2

show much finer structures in the dissipation rate field of case II, indicating generation of more small scales due to the increase in the Reynolds number. In paper [18], we observed how the combustion heat release could damp the small scales and merge them to larger structures. Here, with increasing the Reynolds number the trend is clearly the opposite and the structures are getting finer.

Another important point that can be deduced from Fig. 9 is that with increasing the Reynolds number, the magnitude of the scalar dissipation rate seems to be reduced, this can be illustrated more clearly by plotting the average values in Fig. 10d. However, the scalar dissipation rate has the dimension of an inverse time and is representative of the small-scale mixing. Thus, when it decreases, the corresponding time scale would increase. This will be discussed in more detail under the explanation of Fig. 10d in association with the reduced fuel consumption rates seen in Fig. 8.

Different statistics of the reacting and passive scalars are shown in Fig. 10. The crossstream profiles of the scalar concentrations are displayed in Fig. 10a. This plot confirms that for both non-reacting and reacting scalars, the profiles of different cases approximately collapse when outer scaling is used.

As seen in Fig. 10b, the fluctuation level of the scalars is only weakly affected by increasing the Reynolds number. The peaks of the profiles are almost unchanged. This is clearly contrary to the heat-release effects, where the influences were considerably larger; see [18]. 


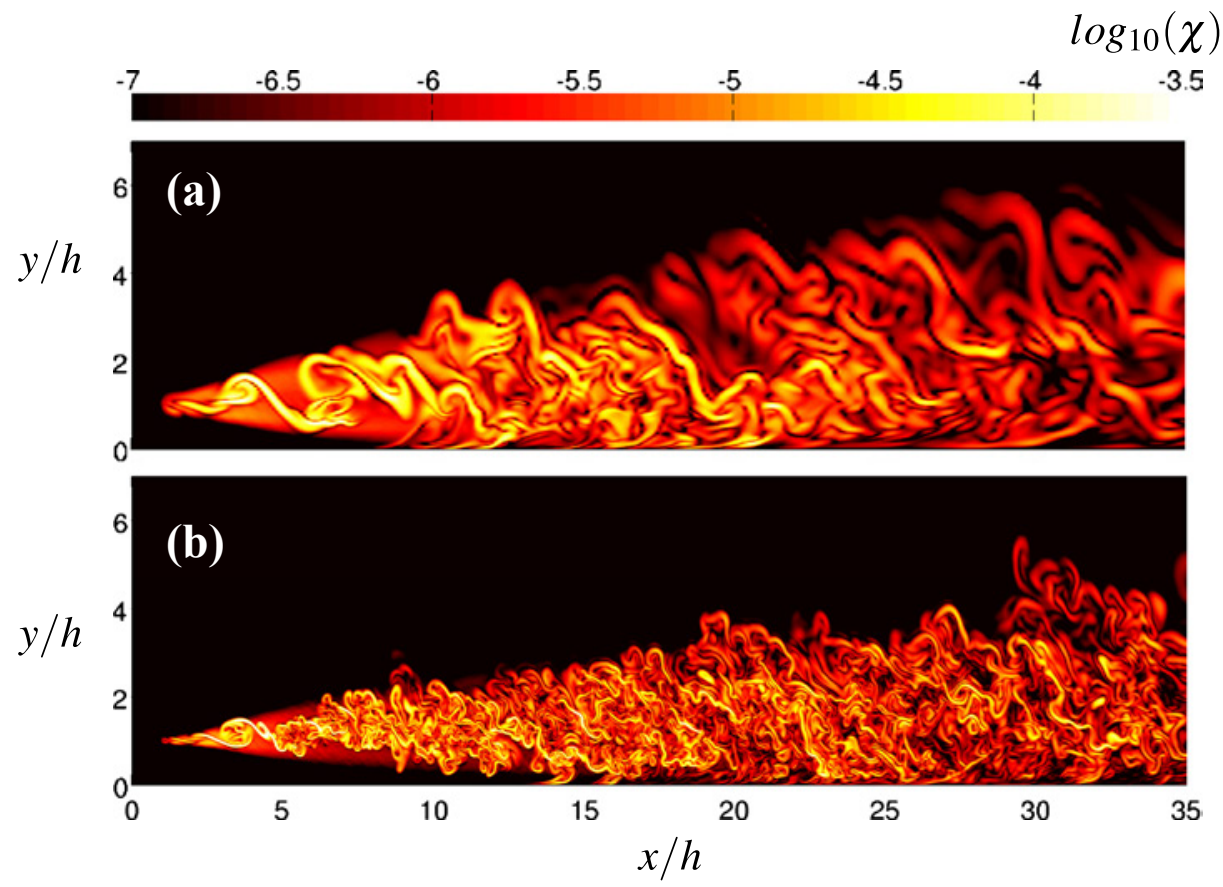

Fig. 9 Iso-contours of the logarithm of the instantaneous scalar dissipation rate, a case I and b caseII

Note that, some of the heat release effects are commonly associated with the changing Reynolds number effects, but this is not the case for the scalar fluctuation intensities. The wall-normal fluxes, shown in Fig. 10c, exhibit a similar behavior.

The mean scalar dissipation rate for both the passive scalar and fuel species are plotted in Fig. 10d. The figure confirms that increasing the Reynolds number decreases the scalar dissipation rate.

From Fig. 10d, we note that the dissipation rates of both passive and reacting scalars, for the lower Reynolds number case, have high values close to the wall, around $y^{+}=10$, where the concentrations are high and the gradients are sharp. However, for the higher Reynolds number case II, the peak of the scalar dissipation rate in the near-wall region is smeared out.

We observed in Fig. 9 that increasing the Reynolds number not only makes the scalar dissipation structures finer but also the magnitude of this quantity changes in total, as observed here in Fig. 10d. The scalar dissipation rate also acts as an inverse of the scalar time scale, thus a decrease corresponds to an increased time scale. This reduction in the local mixing rate may serve as an explanation for the mechanism, underlying the reduction in fuel consumption.

In order to explain, how the total fuel consumption decreases for case II compared to case I, as observed in Fig. 8a, we may consider different parameters that are involved in this reacting flow from a slightly different angle. It is known that in a non-premixed situation, both the chemical reaction timescale and the turbulent mixing influence the reaction rate. In these two cases, the constant global Damköhler numbers are equal; see Eq. 5. However, case II shows a lower scalar dissipation rate compared to case I, meaning a larger mixing time scale. In addition, the length scale has also decreased, as shown in Figs. 1 and $8 \mathrm{~b}$. 

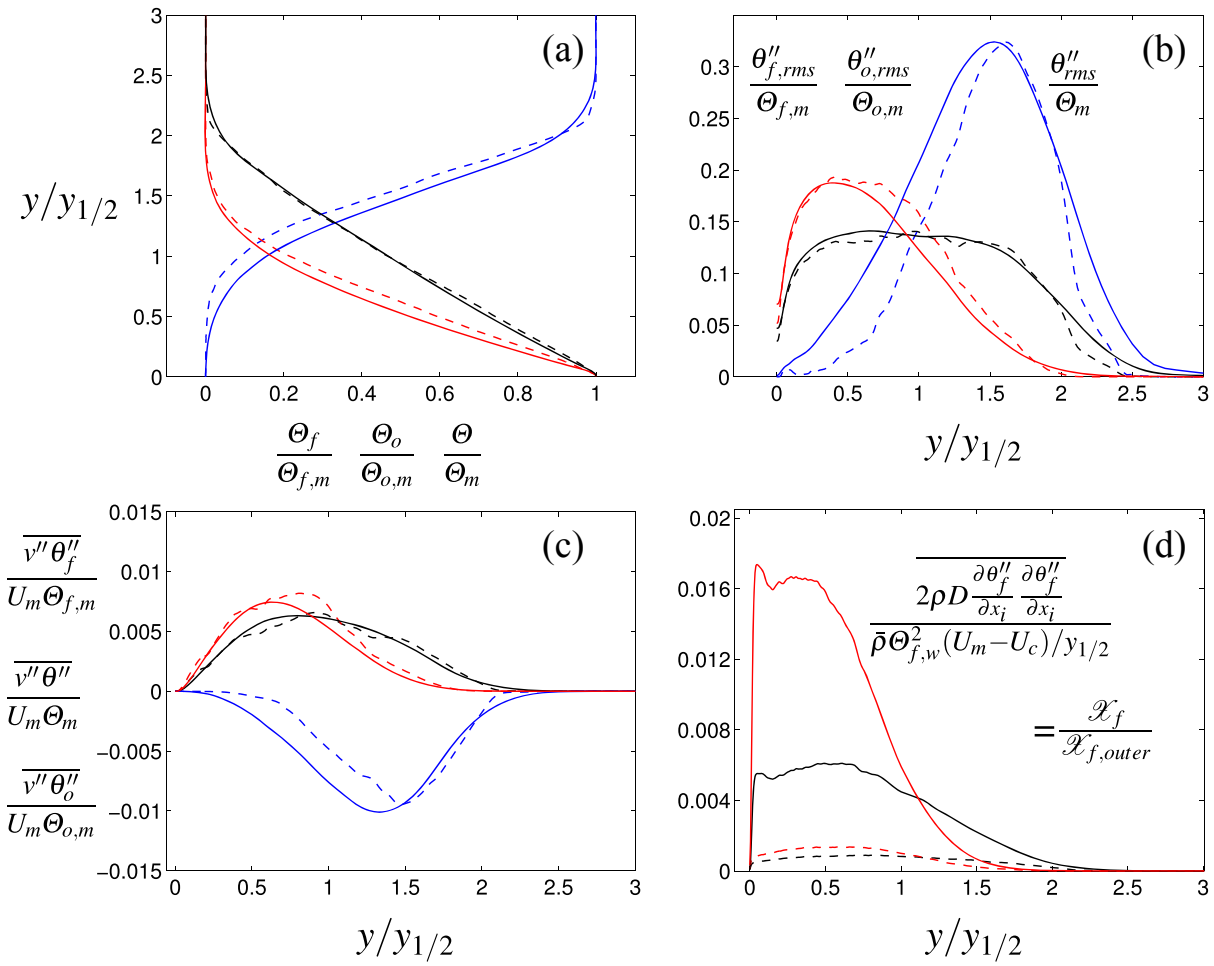

Fig. 10 a Cross-stream profiles of the mean values, $\mathbf{b}$ fluctuation intensity and $\mathbf{c}$ wall-normal fluxes of the scalars; oxidizer: blue, fuel: red, passive: black; Subscript $m$ refers to the maximum value. d Passive (black) and reacting (red) scalar dissipations rates $\chi$ and $\chi_{f}$. Solid line is used for case I and dashed line for case II; Profiles are at $x / h=25$

Considering that the numerical Damköhler number remains a constant in both cases, when the scalar dissipation rate decreases and the corresponding mixing time scale increases, for the global Damköhler number to be a constant, the numerically imposed chemical time scale needs to be increased, which means a decreased reaction rate and thus a reduced fuel consumption for case II.

In turbulent scalar mixing problems, the scalar dissipation rate is known to first increase, because of the appearance of scalar gradients at the largest scales of the turbulence and this is followed by a decrease of scalar dissipation rate after most of large scale fluctuations have vanished. In other words, when the mixtures evolve towards the well-mixed asymptotic case, this is what is observed in these DNS, in case II due to the higher Reynolds number and turbulence intensities, as observed in Fig. 3a-d, the scalar dissipation rate is lower because the reactants undergo a rapid mixing. Hence, the more homogeneous scalar distribution corresponds to a less reactive interface and thus the lower burning rates.

\section{Summary and Conclusions}

Two DNS databases are used to study the Reynolds number effects in turbulent wall-jet flow. The higher Reynolds number simulation case, presented for the first time here, uses about 
180 million cells and is well resolved. The characteristics of turbulent non-premixed walljet flames were studied at these two different Reynolds numbers. Key quantities such as the scalar dissipation rate, reaction rates and the turbulent kinetic energy profiles are discussed thoroughly. The discussion is complemented by illustrating the Reynolds number effects on the two-dimensional premultiplied spectra. Considering both the Taylor micro scale and the Kolmogorov scale as well as the half-height of the wall-jet, the effects of increasing the Reynolds number on wide range of length scales are recognized. In light of the knowledge gained from the changes in different length and time scales, detailed description of how the reaction would differ for the higher Reynolds number case compared to the lower Reynolds number case is provided.

Acknowledgments Computer time provided by Swedish National Infrastructure for Computing (SNIC) is gratefully acknowledged. The financial support from the Swedish National Research program of the Centre for Combustion Science and Technology (CECOST) is gratefully acknowledged.

\section{References}

1. Ahlman, D., Velter, G., Brethouwer, G., Johansson, A.V.: Direct numerical simulation of non-isothermal turbulent wall-jets. Phys. Fluids 21(035), 101 (2009)

2. Bailey, S., Hultmark, M., Monty, J., Alfredsson, P., Chong, M., Duncan, R., Fransson, J.H.M., Hutchins, N., Marusic, I., McKeon, B.J., Nagib, H., Örlü, R., Segalini, A.S., Smits, A.J., Vinuesa, R.: Obtaining accurate mean velocity measurements in high Reynolds number turbulent boundary layers using pitot probes. J. Fluid Mech. 715(1), 642-670 (2013)

3. Cabot, W.H., Cook, A.W.: Reynolds number effects on Rayleigh-Taylor instability with possible implications for type Ia supernovae. Nat. Phys. 2, 562-568 (2006)

4. Chakraborty, N., Swaminathan, N.: Reynolds number effects on scalar dissipation rate transport and its modeling in turbulent premixed combustion. Combust. Sci. Technol. 185(4), 676-709 (2013)

5. DeGraaff, D.B., Webster, D.R., Eaton, J.K.: The effect of Reynolds number on boundary layer turbulence. Exp. Thermal Fluid Sci. 18(4), 341-346 (1998)

6. del Álamo, J.C., Jiménez, J.: Spectra of the very large anisotropic scales in turbulent channels. Phys. Fluids 15(6), 41-44 (2003)

7. Den Toonder, J.M.J., Nieuwstadt, F.T.M.: Reynolds number effects in a turbulent pipe flow for low to moderate Re. Phys. Fluids 9, 3398 (1997)

8. Ferchichi, M., Tavoularis, S.: Reynolds number effects on the fine structure of uniformly sheared turbulence. Phys. Fluids 12, 2942 (2000)

9. Fischer, M., Jovanovic, J., Durst, F.: Reynolds number effects in the near-wall region of turbulent channel flows. Phys. Fluids 13(6), 1755-1767 (2001)

10. Hawkes, E.R., Sankaran, R., Sutherland, J.C., Chen J.H.: Scalar mixing in direct numerical simulations of temporally evolving plane jet flames with skeletal co/h2 kinetics. Proc. Combust. Inst. 31, 1633-1640 (2007)

11. Heinz, S., Roekaerts, D.: Reynolds number effects on mixing and reaction in a turbulent pipe flow. Chem. Eng. Sci. 56(10), 3197-3210 (2001)

12. Hong, J., Katz, J., Schultz, M.P.: Near-wall turbulence statistics and flow structures over threedimensional roughness in a turbulent channel flow. J. Fluid Mech. 667(1), 1-37 (2011)

13. Hoyas, S., Jiménez, J.: Reynolds number effects on the Reynolds-stress budgets in turbulent channels. Phys. Fluids 20(101), 511 (2008)

14. Launder, B.E., Rodi, W.: The turbulent wall jet. Prog. Aerosp. Sci. 19, 81-128 (1981)

15. Peters, N.: Turbulent Combustion. Cambridge Press, Cambridge (2000)

16. Pouransari, Z., Brethouwer, G., Johansson, A.V.: Direct numerical simulation of an isothermal reacting turbulent wall-jet. Phys. Fluids 23(085104) (2011)

17. Pouransari, Z., Johansson, A.V.: Numerical investigation of wall heat transfer in turbulent reacting walljets. In: Tavoularis, S. (ed.) Proceedings of the Seventh International Symposium on Turbulence and Shear Flow Phenomena. Ottawa (2011)

18. Pouransari, Z., Vervisch, L., Johansson, A.: Heat release effects on mixing scales of non-premixed turbulent wall-jets: a DNS study. Int. J. Heat Fluid Flows 40, 65-80 (2013) 
19. Rasam, A., Brethouwer, G., Johansson, A.: An explicit algebraic model for the subgridscale passive scalar flux. J Fluid Mech 721, 541-577 (2013)

20. Rasam, A., Brethouwer, G., Schlatter P, Li, Q., Johansson, A.: Effects of modelling, resolution and anisotropy of subgrid-scales on large eddy simulations of channel flow. J. Turbul. 12, N10 (2011). doi:10.1080/14685248.2010.541920

21. Saikrishnan, N., Angelis, E., Longmire, E.K., Marusic, I., Casciola, C.M., Piva, R.: Reynolds number effects on scale energy balance in wall turbulence. Phys. Fluids 24(015), 101 (2012)

22. Schumacher, J.: Reynolds number effects on the turbulent mixing of passive scalars. In: IUTAM Symposium on Computational Physics and New Perspectives in Turbulence, pp. 85-90. Springer (2008)

23. Vervisch, L., Poinsot, T.: Direct numerical simulation of non-premixed turbulent flames. Ann. Rev. Fluid Mech. 30, 655-691 (1998)

24. Wei, T., Willmarth, W.: Reynolds-number effects on the structure of a turbulent channel flow. J. Fluid Mech. 204, 57-95 (1989)

25. Yiu, M.W., Zhou, Y., Zhou, T., Cheng, L.: Reynolds number effects on three-dimensional vorticity in a turbulent wake. AIAA J. 42(5), 1009-1016 (2004) 